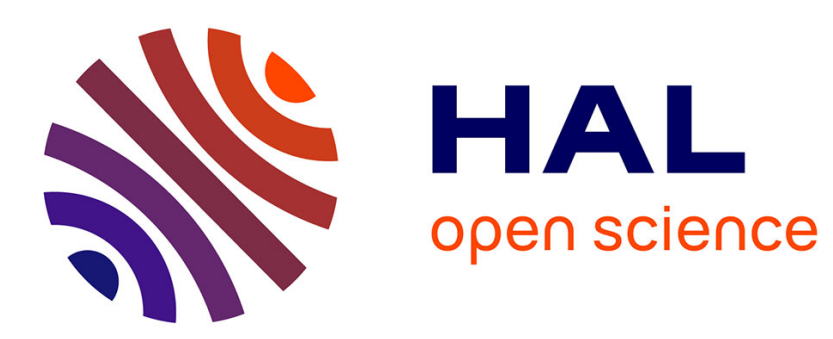

\title{
Divergence, Hopf and double-zero bifurcations of a nonlinear planar beam
}

\author{
Angelo Luongo, Angelo Di Egidio
}

\section{To cite this version:}

Angelo Luongo, Angelo Di Egidio. Divergence, Hopf and double-zero bifurcations of a nonlinear planar beam. Computers \& Structures, 2006, 84 (24-25), pp.1596-1605. hal-00790771

\section{HAL Id: hal-00790771 https://hal.science/hal-00790771}

Submitted on 21 Feb 2013

HAL is a multi-disciplinary open access archive for the deposit and dissemination of scientific research documents, whether they are published or not. The documents may come from teaching and research institutions in France or abroad, or from public or private research centers.
L'archive ouverte pluridisciplinaire HAL, est destinée au dépôt et à la diffusion de documents scientifiques de niveau recherche, publiés ou non, émanant des établissements d'enseignement et de recherche français ou étrangers, des laboratoires publics ou privés. 


\title{
Divergence, Hopf and double-zero bifurcations of a nonlinear planar beam
}

\author{
Angelo Luongo *, Angelo Di Egidio ${ }^{1}$ \\ Dipartimento di Ingegneria delle Strutture delle Acque e del Terreno, Università di L'Aquila, 67040 Monteluco di Roio, L'Aquila, Italy
}

\begin{abstract}
The multiple scale method is directly applied to a one dimensional continuous model to derive the equations governing the system asymptotic dynamic around a bifurcation point. The theory is illustrated with reference to a specific example, namely an internally con strained planar beam, equipped with a lumped visco elastic device and loaded by a follower force. Nonlinear, integro differential equa tions of motion are derived and expanded upto cubic terms in the transversal displacements and velocities of the beam. The linear stability of the trivial equilibrium is first studied. It reveals the existence of divergence and Hopf and double zero bifurcations. The spec tral properties of the linear operator are studied at the bifurcation points by obtaining closed form expressions. Notably, the system pos ses an incomplete system of eigenvectors at the double zero point (i.e. it is defective or nilpotent), thus entailing the need to find a generalized eigenvector. A multiple scale analysis is then performed for the three bifurcations and the relevant bifurcation equations are derived directly in their normal forms. Finally, they are numerically studied to furnish a comprehensive scenario of the postcritical behaviour around the bifurcations.
\end{abstract}

Keywords: Bifurcation; Multiple scales method; Double zero eigenvalue; Divergence; Hopf; Beam continuous model

\section{Introduction}

Reduction methods play a fundamental role in nonlinear dynamics. They allow the essential dynamics of the original system to be captured using models with a very low number of degrees of freedoms, thus avoiding the use of brutal numerical modelling.

Reduction methods have been thoroughly discussed by Steindl and Troger [1], who compared linear and nonlinear Galerkin methods, the center manifold and the approximate inertial manifold theories. Nayfeh [2] also developed a modified version of the Galerkin method, able to overcome the shortcomings of the classical procedure. Nayfeh and co-workers $[3,4]$ have also widely used the multiple

\footnotetext{
* Corresponding author. Tel.: +390862 434521; fax: +390862434548.

E mail addresses: luongo@ing.univaq.it (A. Luongo), ade@ing. univaq.it (A.D. Egidio).

${ }^{1}$ Tel.: + 390862434530 .
}

scale method in the so-called direct form, i.e. by dealing with the partial differential equations and boundary conditions rather than with algebraic systems obtained by means of a priori discretization. Within the framework of bifurcation theory, the authors have systematically applied the multiple scale method to finite-dimensional systems to derive the relevant bifurcation equations [5 7]. The method make it possible to avoid both the search for the center manifold and the use of the normal form theory, since the algorithm furnishes bifurcation equations directly in normal form.

In a companion paper [8], an attempt has been made by the authors to extend the method to infinite-dimensional systems. A key point of the procedure lies in defining a scalar product and using the bilinear identity to get the linear adjoint problem. The solution of the relevant homogeneous problem supplies the tool to enforce the solvability conditions at any order of the perturbation procedure. After reconstitution [9], these equations furnish the desired 
bifurcation equations. Here, the main steps of the procedure are resumed in a more problem-oriented form and some new results presented. A combined analytical and numerical analysis is performed to investigate the mechanical behaviour of the system close to the bifurcation.

\section{Model}

A planar, inextensible and shear-undeformable straight beam is considered, fixed at end $A$, constrained by a linear viscous-elastic device at end $B$ and loaded by a follower force $P$ at $B$ (Fig. 1). The device consists of an extensional spring of stiffness $k_{\mathrm{e}}$ and two dashpots, of constants $c_{\mathrm{e}}$ and $c_{\mathrm{t}}$, of an extensional and a torsional type, respectively.

The actual configuration of the beam is described by the transversal displacement field $\tilde{u}(\tilde{s}, t)$, the longitudinal displacement $\tilde{w}(\tilde{s}, t)$ and the rotation $\tilde{\vartheta}(\tilde{s}, t)$, where $\tilde{s} \in[0, l]$ is an abscissa. The three displacements, however, are not independent, because of the internal constraints:

$\sin \tilde{\vartheta}=\tilde{u^{\prime}}$

$\varepsilon:=\sqrt{ }\left(1+\tilde{w}^{\prime}\right)^{2}+\tilde{u^{\prime}}-1=0$

expressing shear-undeformability and inextensibility, respectively. The curvature $\varsigma(\tilde{s}, t)$ is assumed as the (unique) strain measure; from Eq. $\left(1_{1}\right)$ it follows, that

$\varsigma:=\tilde{\vartheta^{\prime}}=\tilde{u^{\prime \prime}} / \sqrt{ } 1-\tilde{u^{\prime 2}}$

The equations of motion are derived by the generalized Hamiltonian principle, introducing the constraint equation $\left(1_{2}\right)$ by a Lagrangian multiplier $\tilde{\lambda}(\tilde{s}, t)$, having the meaning of axial (reactive) force. By using Eq. $\left(1_{1}\right)$ to eliminate the rotation $\tilde{\vartheta}(\tilde{s}, t)$ and expanding $\tilde{u}(\tilde{s}, t)$ in Taylor series, the equations of motion, corrected up to the third-order, are derived.

The longitudinal displacement $\tilde{w}(\tilde{s}, t)$ and the axial force $\tilde{\lambda}(\tilde{s}, t)$ are then condensed by integrating the relevant equation of motion. Finally, the following equations and boundary conditions in the unique variable $\tilde{u}(\tilde{s}, t)$ are derived. By introducing the following nondimensional parameters:

$$
\begin{aligned}
& \tau=\omega t ; \quad s=\tilde{s} / l ; \quad u=\tilde{u} / l ; \quad w=\tilde{w} / l ; \\
& \omega^{2}=E I / m l^{4} ; \quad 2 \mu=P l^{2} / E I ; \quad \lambda=\lambda / m l^{2} \omega^{2} ; \\
& \kappa=k_{\mathrm{e}} l^{3} / E I ; \quad \xi_{\mathrm{e}}=c_{\mathrm{e}} \omega l^{3} / E I ; \quad \xi_{\mathrm{t}}=c_{\mathrm{t}} \omega l / E I
\end{aligned}
$$

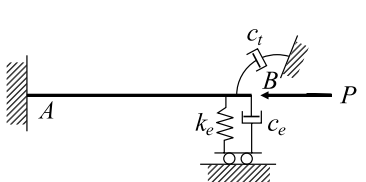

(a)

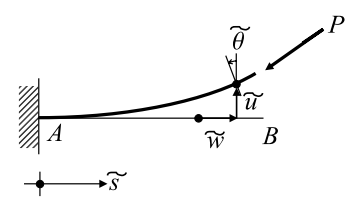

(b)
Fig. 1. Model and displacements. where $\omega$ is a frequency, $m$ the mass for length unity and $E I$ the flexural stiffness of the beam, the nondimensional equations of motion and the relevant boundary conditions read:

$$
\begin{aligned}
& \ddot{u}+u^{\mathrm{IV}}+\left[u^{\prime}\left(u^{\prime} u^{\prime \prime}\right)^{\prime}\right]^{\prime}+2 \mu\left(1-u_{B}^{\prime 2} / 2\right) u^{\prime \prime}+\left[\int_{1}^{s}(\right. \\
& +\left[\left(\int_{0}^{s} u^{\prime 2} / 2 \mathrm{~d} s\right)\right] u^{\prime}=0 \\
& -\left(u_{B}^{\prime \prime \prime}+u_{B}^{\prime \prime \prime} u_{B}^{\prime 2}+u_{B}^{\prime \prime 2} u_{B}^{\prime}\right)+\kappa u_{B}+\xi_{\mathrm{e}} \dot{u}_{B}+\mu u_{B}^{\prime 3}=0 \\
& \left(u_{B}^{\prime \prime}+u_{B}^{\prime \prime} u_{B}^{\prime 2}\right)+\xi_{\mathrm{t}}\left(\dot{u}_{B}^{\prime}+2 u_{B}^{\prime 2} \dot{u}_{B}^{\prime}\right)=0 \\
& u_{A}=0, \quad u_{A}^{\prime}=0
\end{aligned}
$$

where $A$ and $B$ denote evaluation at the beam ends.

It should be noted that the nonlinear viscous term in the bending moment boundary condition $(4 \mathrm{c})$ arises from the expansion of the rotation $\vartheta_{B}$ in terms of the transversal displacement $u_{B}$ (Eq. (1a)). A similar nonlinearity in the geometrical boundary conditions (4d) has, in contrast, been omitted, since $\vartheta_{A}$ vanishes when its linear part vanishes.

\section{Linear stability boundaries}

The linearized problem (4) is first studied. By letting $u(s) \quad \phi(s) \exp (\lambda t)$, the following eigenvalue problem is obtained:

$$
\begin{aligned}
& f(s):=\phi^{\mathrm{IV}}+2 \mu \phi^{\prime \prime}+\lambda^{2} \phi=0 \\
& T_{B}:=-\phi_{B}^{\prime \prime \prime}+\kappa \phi_{B}+\lambda \xi_{\mathrm{e}} \phi_{B}=0 \\
& M_{B}:=\phi_{B}^{\prime \prime}+\lambda \xi_{\mathrm{t}} \phi_{B}^{\prime}=0 \\
& \phi_{A}=0, \quad \phi_{A}^{\prime}=0
\end{aligned}
$$

The parameters $\mu$ and $\kappa$ are taken as bifurcation parameters and the boundaries at which the trivial equilibrium position loses stability are sought in the $(\kappa, \mu)$ parameter plane. Eq. (5a) admits the following solution, accounting for the geometrical boundary conditions (5d) at $A$ :

$\phi(s)=c_{1}(\cos p s-\cosh q s)+c_{2}\left(\sin p s-\frac{p}{q} \sinh q s\right)$

where $c_{1}$ and $c_{2}$ are arbitrary constants and

$q^{2}:=\sqrt{\mu^{2}-\lambda^{2}-\mu}$
$p^{2}:=\sqrt{\mu^{2}-\lambda^{2}+\mu}$

have been posed. With Eq. (6) the mechanical boundary conditions $((5 \mathrm{~b})$ and $(5 \mathrm{c}))$ at $B$ read:

$\mathbf{R}(\lambda, \kappa, \mu) \mathbf{c}=\mathbf{0}$

with $\mathbf{R}$ a $2 \times 2$ matrix. By vanishing the determinant of $\mathbf{R}$ a transcendent equation in $\lambda$ follows, depending on the bifurcation parameters; it is analyzed below.

\subsection{Divergence boundary and double-zero point}

When $\lambda \rightarrow 0$, by expanding Eqs. ((7a) and (7b)) in Mc Laurin series, $p^{2} 2 \mu+\mathrm{O}\left(\lambda^{2}\right), q^{2}-\lambda^{2} / 2 \mu+\mathrm{O}\left(\lambda^{3}\right)$ follow, from which the first-order approximations are 


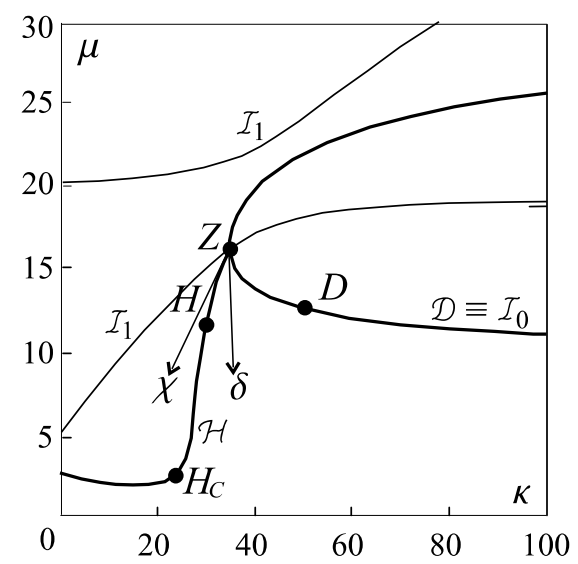

Fig. 2. Evaluation of the double zero point $Z$ : curves $I_{k}$ denote vanishing of the invariants $I_{k}$ of the characteristic equation; $\xi_{\mathrm{t}}=0.05, \xi_{\mathrm{e}}=0.025$.

obtained: $p \cong \sqrt{ } 2 \mu$ and $q \cong \mathrm{i} \lambda / \sqrt{ } 2 \mu$. By substituting them in $\mathbf{R}$ and expanding its determinant in series of $\lambda$, it follows:

$\operatorname{det} \mathbf{R}(\lambda, \kappa, \mu)=I_{0}+\lambda I_{1}+\lambda^{2} I_{2}+\cdots=0$

with $I_{j} \quad I_{j}(\kappa, \mu)$ as the invariant of the system. In particular, the leading invariants are found to be:

$I_{0}=2.82 \mu^{3 / 2} \kappa \cos (\sqrt{ } 2 \mu) \quad 5.66 \mu^{5 / 2} \quad 2 \mu \kappa \sin (\sqrt{ } 2 \mu)$

$I_{1}=2[\sqrt{ } 2 \mu(\mu \alpha+\kappa) \cos (\sqrt{ } 2 \mu) \quad \kappa \sqrt{ } 2 \mu \quad \mu(2 \mu+\alpha \quad \kappa) \sin (\sqrt{ } 2 \mu)]$

where $\alpha:=\xi_{\mathrm{e}} / \xi_{\mathrm{t}}$ has been posed. The divergence boundary $\mathcal{D}$ has codimension- 1 and is defined by the equation $I_{0}(\kappa, \mu) \quad 0$. The double-zero point has codimension-2 and is determined by the simultaneous vanishing of the first two invariants, $I_{0}(\kappa, \mu) \quad 0$ and $I_{1}(\kappa, \mu) \quad 0$; therefore, it depends on the damping ratio $\alpha$ only, not on the values of the single coefficients $\xi_{\mathrm{e}}$ and $\xi_{\mathrm{t}}$. The divergence curve $\mathcal{D} \equiv I_{0}$ and the double-zero point $Z \equiv I_{0} \cap I_{1}$ are displayed in Fig. 2 for $\alpha \quad 0.5$.

\subsection{Hopf boundary}

If $\lambda \quad \mathrm{i} \omega$, then $p$ and $q$ are real (Eq. (7)). By substituting them in matrix $\mathbf{R}$ and separating the real and imaginary parts of $\operatorname{det}(\mathbf{R})$, two real equations are obtained, namely $\mathcal{H}_{i}(\mu, \kappa, \omega)=0$ (i 1,2). They define a codimension-1 curve $\mathrm{H}$ in the bifurcation parameter plane (Fig. 2), depending both on $\xi_{\mathrm{e}}$ and $\xi_{\mathrm{t}}$ coefficients. According to the Takens Bogdanova bifurcation mechanism, the Hopf boundary dies when collides with the divergence boundary at the double-zero point $Z$.

\section{The adjoint problem}

It is useful, for future use, to derive the adjoint problem to the linearized direct problem (5). To define it, an inner product must be first introduced. Eqs. (5) associate to any admissible function $\phi(s)$ satisfying the geometrical boundary conditions (5d), a system of forces $\mathbf{f}:=\left\{f(s), T_{B}, M_{B}\right\}$, where $f(s)$ are body forces acting in the domain, and $T_{B}$ and $M_{B}$ are a shear force and a bending moment acting at $B$. Eqs. (5), in operator form, $\operatorname{read} \mathbf{f}:=\mathbf{L} \boldsymbol{\Phi} \quad \mathbf{0}$ where $\boldsymbol{\Phi}:=\left\{\phi(s), \phi_{B}, \phi_{B}^{\prime}\right\}$. Dual variables $\boldsymbol{\Psi}:=\left\{\psi(s), \psi_{B}, \psi_{B}^{\prime}\right\}$ are considered, having the meaning of a virtual displacements field $\psi(s)$, together with its value and its first derivative at $B$. A scalar product is then defined, as the virtual work done by the forces $\mathbf{f}$ in the virtual displacements $\boldsymbol{\Psi}$ :

$$
(\boldsymbol{\Psi}, \mathbf{f}):=\int_{0}^{1} \psi(s) f(s) \mathrm{d} s+\psi_{B} T_{B}+\psi^{\prime}{ }_{B} M_{B}
$$

where the overbar denotes complex conjugate. Finally, from the bilinear identity:

$$
(\boldsymbol{\Psi}, \mathbf{L} \boldsymbol{\Phi})=\left(\mathbf{L}^{*} \boldsymbol{\Psi}, \boldsymbol{\Phi}\right)
$$

the adjoint problem $\mathbf{L}^{*} \boldsymbol{\Psi} \mathbf{0}$ follows. By using Eqs. (5) in Eq. (11) and performing integration by parts according to Eq. (12), the adjoint field equation and adjoint boundary conditions read:

$$
\begin{aligned}
& \psi^{\mathrm{IV}}+2 \mu \psi^{\prime \prime}+\lambda^{2} \psi=0 \\
& -\psi_{B}^{\prime \prime \prime}+\kappa \psi_{B}-2 \mu \psi_{B}^{\prime}+\lambda \xi_{\mathrm{e}} \psi_{B}=0 \\
& \psi_{B}^{\prime \prime}+2 \mu \psi_{B}+\lambda \xi_{\mathrm{t}} \psi_{B}^{\prime}=0 \\
& \psi_{A}=0, \quad \psi_{A}^{\prime}=0
\end{aligned}
$$

\section{Bifurcation analysis}

The multiple scale method is applied to analyze the system's behaviour around a divergence point (say $D$, in Fig. 2), a Hopf point (say $H$ ) and the double-zero point $(Z)$. A perturbation parameter $\varepsilon$ is introduced as a measure of the distance of a given point from the bifurcation point in the parameter space. Different $\varepsilon$-dependent time-scales $t_{k} \quad \varepsilon^{k} t$ are defined and the state variable $u(s)$ are expanded in Taylor series of $\varepsilon$. By equating terms of the same power of $\varepsilon$, linear perturbation equations having the same operator are obtained, and then solved in sequence for the series coefficients. Except for the lower-order eigenvalue problem, higher-order equations are nonhomogeneous; they admit a solution if and only if the known term belongs to the range of the singular operator, i.e. if it is orthogonal to the solutions of the adjoint homogeneous problem (13). Solvability equations, when combined on the true timescale $t$ (according to the so-called reconstitution method), furnish the bifurcation equations, governing the asymptotic dynamics of the system, reduced to the center manifold. The procedure is sketched in the following, for codimension-1 and codimension-2 bifurcations.

\subsection{Codimension-1 bifurcation}

The load $\mu$ is taken as bifurcation parameter, and its deviation from the bifurcation value $\mu_{0}$ denoted by $\gamma:=\mu-\mu_{0} \quad \mathrm{O}\left(\varepsilon^{2}\right)$. By introducing the series expansions:

$u=\varepsilon u_{1}+\varepsilon^{3} u_{3}+\cdots$

$\mathrm{d} / \mathrm{d} t=d_{0}+\varepsilon^{2} d_{2}+\cdots$ 


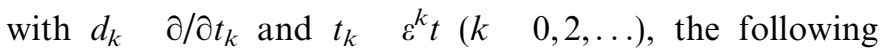
perturbation equations and boundary conditions are derived:

$$
\begin{aligned}
\varepsilon: & d_{0}^{2} u_{1}+u_{1}^{\mathrm{IV}}+2 \mu_{0} u_{1}^{\prime \prime}=0 \\
& \xi_{\mathrm{e}} d_{0} u_{1 B}-u_{1 B}^{\prime \prime \prime}+\kappa u_{1 B}=0 \\
& \xi_{\mathrm{t}} d_{0} u_{1 B}^{\prime}+u_{1 B}^{\prime \prime}=0 \\
& u_{1 A}=0, \quad u_{1 A}^{\prime}=0 \\
\varepsilon^{3}: & d_{0}^{2} u_{3}+u_{3}^{\mathrm{IV}}+2 \mu_{0} u_{3}^{\prime \prime}=-2 d_{0} d_{2} u_{1}-2 \gamma u_{1}^{\prime \prime}+n_{1}\left(u_{1}\right) \\
& \xi_{\mathrm{e}} d_{0} u_{3 B}-u_{3 B}^{\prime \prime \prime}+\kappa u_{3 B}=-\xi_{\mathrm{e}} d_{2} u_{1 B}+n_{2}\left(u_{1}\right) \\
& \xi_{\mathrm{t}} d_{0} u_{3 B}^{\prime}+u_{3 B}^{\prime \prime}=-\xi_{\mathrm{t}} d_{2} u_{1 B}^{\prime}+n_{3}\left(u_{1}\right) \\
& u_{3 A}=0, \quad u_{3 A}^{\prime}=0
\end{aligned}
$$

where $n_{1}, n_{2}$ and $n_{3}$ are the nonlinear terms in Eqs. (4a) (4c) evaluated at $u \quad u_{1}$. Eq. (15a) admit the steady solutions:

$u_{1}(s)=a\left(t_{2}, t_{4}, \ldots\right) \phi(s) \quad$ or

$u_{1}(s)=A\left(t_{2}, t_{4}, \ldots\right) \mathrm{e}^{\mathrm{i} \omega t_{0}} \phi(s)+$ c.c.

at the divergence or Hopf point, respectively. In Eqs. (16) $a$ (or $A$ ) is a real (or complex) amplitude depending on the slower time-scales; moreover $\phi(s)$ is a real (or complex) eigenvector. By substituting Eqs. (16) in Eq. (15b) and enforcing solvability, the bifurcation equations are drawn for the two cases:

$\dot{a}+c_{1 \gamma} a \gamma+c_{3} a^{3}=0$ or

$\dot{A}+C_{1 \gamma} A \gamma+C_{3} A^{2} \bar{A}=0$

where $c_{i}$ and $C_{i}$ are coefficients (see Appendix).

\subsection{Codimension-2 bifurcation}

The load $\mu$ and the stiffness $\kappa$ are taken as bifurcation parameters, and their increments with respect to the critical values $\mu_{0}$ and $\kappa_{0}$, denoted by:

$\gamma:=\mu-\mu_{0}=\mathrm{O}\left(\varepsilon^{2}\right)$

$\beta:=\kappa-\kappa_{0}=\mathrm{O}\left(\varepsilon^{2}\right)$

Expansion of the variables is less straightforward. Since the linear operator is defective (i.e. the set of eigenvectors is not complete at the bifurcation) fractional power expansions should be employed, according to the treatment of the algebraic systems. Such expansions lead to a cascade of perturbation equations in which nonlinearities appear only at a higher-order. Solution of the lower-order linear equations gives rise the so-called generalized eigenvectors, which complete the defective base [10]. When the eigenvalue multiplicity is equal to 2, the appropriate expansion for a generic system contains powers of $\varepsilon^{1 / 2}$. However, due to the fact the system under study posses only odd nonlinearities, only even powers of $\varepsilon^{1 / 2}$ are meaningful since odd powers would furnish trivial equations; therefore a standard series expansion of $\varepsilon$ is recovered. Notably, when a defective bifurcation is analyzed via a perturbation method, the first-order form of the equations of motion is more convenient, since the generalized eigenvector theory is also stated in this form. Therefore, by recasting the equations of motion by letting $v:=\mathrm{d} u / \mathrm{d} t$, perturbation equations of the following type are obtained:

$$
\begin{aligned}
\varepsilon: & d_{0} u_{1}-v_{1}=0 \\
& d_{0} u_{1 B}-v_{1 B}=0 \\
& d_{0} u_{1 B}^{\prime}-v_{1 B}^{\prime}=0 \\
& d_{0} v_{1}+u_{1}^{\mathrm{IV}}+2 \mu_{0} u_{1}^{\prime \prime}=0 \\
& -u_{1 B}^{\prime \prime \prime}+\kappa_{0} u_{1 B}+\xi_{\mathrm{e}} v_{1 B}=0 \\
& u_{1 B}^{\prime \prime}+\xi_{\mathrm{t}} v_{1 B}^{\prime}=0 \\
& u_{1 A}=0, \quad u_{1 A}^{\prime}=0 \\
\varepsilon^{2}: & d_{0} u_{2}-v_{2}=-d_{1} u_{1} \\
& d_{0} u_{2 B}-v_{2 B}=-d_{1} u_{1 B} \\
& d_{0} u_{2 B}^{\prime}-v_{2 B}^{\prime}=-d_{1} u_{1 B}^{\prime} \\
& d_{0} v_{2}+u_{2}^{\mathrm{IV}}+2 \mu_{0} u_{2}^{\prime \prime}=-d_{1} v_{1} \\
& -u_{2 B}^{\prime \prime \prime}+\kappa_{0} u_{2 B}+\xi_{\mathrm{e}} v_{2 B}=0 \\
& u_{2 B}^{\prime \prime}+\xi_{\mathrm{t}} v_{2 B}^{\prime}=0 \\
& u_{2 A}=0, \quad u_{2 A}^{\prime}=0 \\
\varepsilon^{3}: & d_{0} u_{3}-v_{3}=-d_{2} u_{1}-d_{1} u_{2} \\
& d_{0} u_{3 B}-v_{3 B}=-d_{2} u_{1 B}-d_{1} u_{2 B} \\
& d_{0} u_{3 B}^{\prime}-v_{3 B}^{\prime}=-d_{2} u_{1 B}^{\prime}-d_{1} u_{2 B}^{\prime} \\
& d_{0} v_{3}+u_{3}^{\mathrm{IV}}+2 \mu_{0} u_{3}^{\prime \prime}=-d_{2} v_{1}-d_{1} v_{2}-2 \gamma u_{1}^{\prime \prime}+n_{1}\left(u_{1}^{3}\right) \\
& -u_{3 B}^{\prime \prime \prime}+\kappa_{0} u_{3 B}+\xi_{\mathrm{e}} v_{3 B}=-\beta u_{1 B}+n_{2}\left(u_{1}^{3}\right) \\
& u_{3 B}^{\prime \prime}+\xi_{\mathrm{t}} v_{3 B}^{\prime}=n_{3}\left(u_{1}^{3}\right) \\
& u_{3 A}=0, \quad u_{3 A}^{\prime}=0 \\
\varepsilon^{4}: & d_{0} u_{4}-v_{4}=-d_{3} u_{1}-d_{2} u_{2}-d_{1} u_{3} \\
& d_{0} u_{4 B}-v_{4 B}=-d_{3} u_{1 B}-d_{2} u_{2 B}-d_{1} u_{3 B} \\
& d_{0} u_{4 B}^{\prime}-v_{4 B}^{\prime}=-d_{3} u_{1 B}^{\prime}-d_{2} u_{2 B}^{\prime}-d_{1} u_{3 B}^{\prime} \\
& d_{0} v_{4}+u_{4}^{\mathrm{IV}}+2 \mu_{0} u_{4}^{\prime \prime}=-d_{3} v_{1}-d_{2} v_{2}-d_{1} v_{3} \\
& -2 \gamma u_{2}^{\prime \prime}+n_{1}\left(u_{1}^{2} u_{2}\right) \\
& -u_{4 B}^{\prime \prime \prime}+\kappa_{0} u_{4 B}+\xi_{\mathrm{e}} v_{4 B}=-\beta u_{2 B}+n_{2}\left(u_{1}^{2} u_{2}\right) \\
& u_{4 B}^{\prime \prime}+\xi_{\mathrm{t}} v_{4 B}^{\prime}=n_{3}\left(u_{1}^{2} u_{2}\right) \\
& u_{4 A}=0, \quad u_{4 A}^{\prime}=0 \\
&
\end{aligned}
$$

By solving in chain the perturbation equations, the generalized, order-two, eigenvector naturally appears at the $\varepsilon^{2}$-order as an element of Keldysh chain [11] (which generalizes the concept of Jordan chain valid for an algebraic operator). Such equations do not require any solvability conditions to be solved, since the known term belongs to the range of the operator. However, the first-order derivative of the amplitude, $d_{1} a$, remains undetermined at this order; as a consequence, second-order derivatives $d_{1}^{2} a$ appear at $\varepsilon^{3}$-order, at which meaningful solvability equations firstly appear. They are found to be of the form:

$$
\begin{aligned}
& d_{1}^{2} a=\left(c_{1 \beta} \beta+c_{1 \gamma} \gamma\right) a+c_{3} a^{3} \\
& 2 d_{1} d_{2} a=\left(e_{1 \beta} \beta+e_{1 \gamma} \gamma\right) d_{1} a+e_{3} a^{2} d_{1} a
\end{aligned}
$$




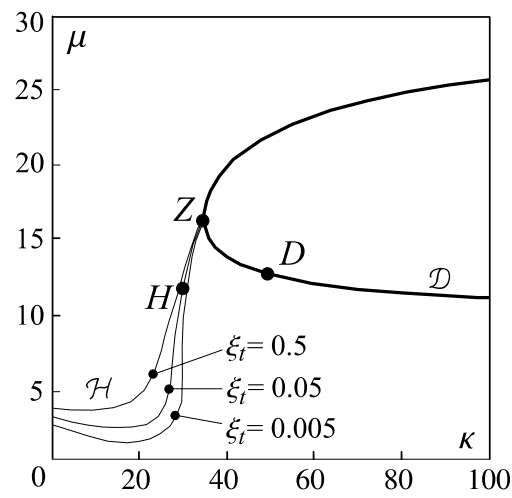

(a)

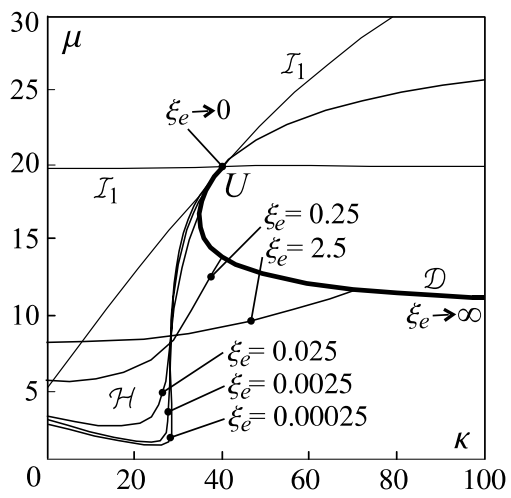

(b)

Fig. 3. Linear stability diagram $\left(\mathcal{D}\right.$ : divergence boundary, $\mathcal{H}$ : Hopf boundary); (a) $\alpha=\xi_{\mathrm{e}} / \xi_{\mathrm{t}}=0.5$; (b) $\xi_{\mathrm{t}}=0.05$.

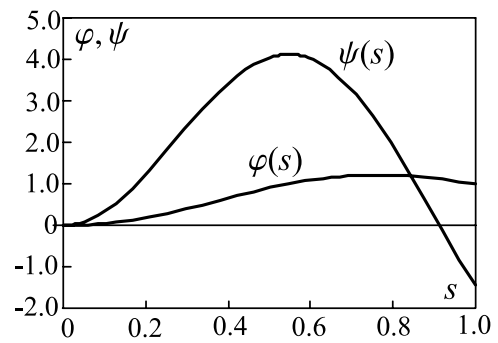

(a)
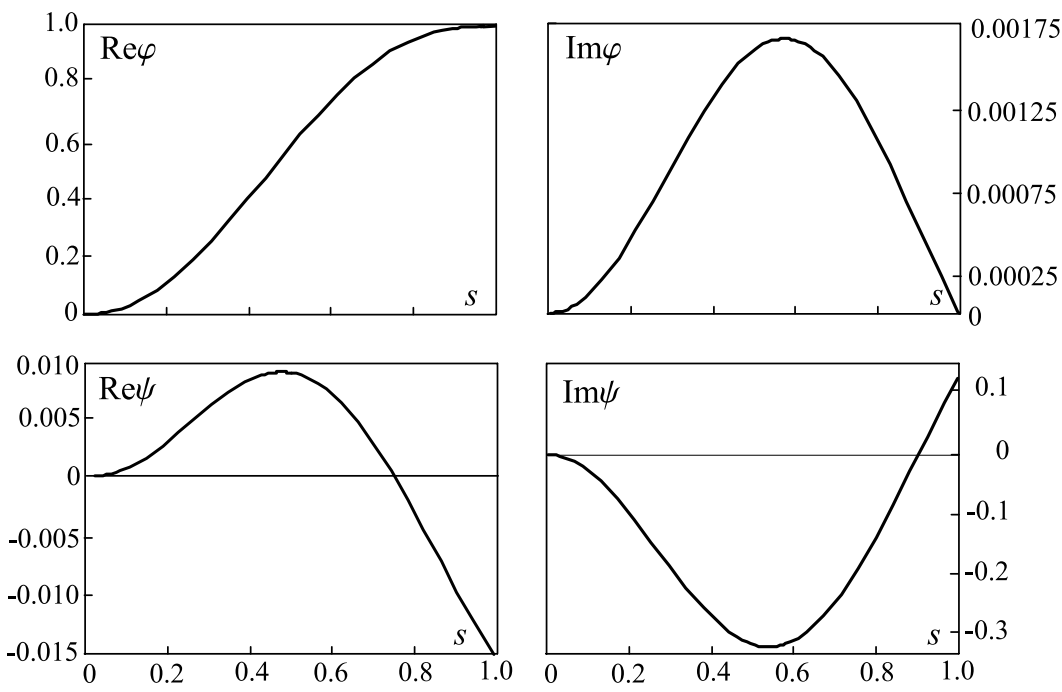

(b)
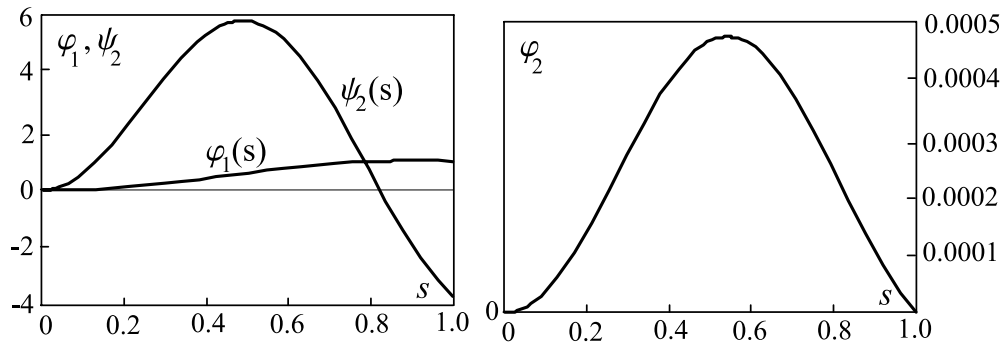

(c)

Fig. 4. Eigenvectors: (a) right $\varphi$ and left $\psi$ eigenvectors at the divergence point $D$; (b) right $\varphi$ and left $\psi$ eigenvectors at the Hopf point $H$; (c) proper (right $\varphi_{1}$ and left $\psi_{2}$ ) and generalized (right $\varphi_{2}$ ) eigenvectors at the double zero point $Z$. 
where $c_{i}$ and $e_{i}$ are real coefficients (see Appendix). By observing that $\mathrm{d} / \mathrm{d} t \quad \varepsilon d_{1}+\varepsilon^{2} d_{2}+\cdots$, it follows that $\mathrm{d}^{2} a / \mathrm{d} t^{2}=\left(\varepsilon d_{1}+\varepsilon^{2} d_{2}+\cdots\right)^{2} a=\left(\varepsilon^{2} d_{1}^{2}+2 \varepsilon^{3} d_{1} d_{2}+\cdots\right) a$; then the solvability conditions $((20 \mathrm{a})$ and $(20 \mathrm{~b}))$ can be combined to obtain an unique differential equation of order 2, namely:

$$
\ddot{a}=\left(c_{1 \beta} \beta+c_{1 \gamma} \gamma\right) a+\left(e_{1 \beta} \beta+e_{1 \gamma} \gamma\right) \dot{a}+c_{3} a^{3}+e_{3} a^{2} \dot{a}
$$

where the $\varepsilon$-parameter has been reabsorbed. Eq. (21) directly appears in the Bogdanova Arnold form.

\section{Numerical results}

\subsection{Linear analysis}

Sensitivity of the Hopf boundaries to modification of the damping coefficients is first studied. If $\xi_{\mathrm{e}}$ and $\xi_{\mathrm{t}}$ are varied by keeping fixed their ratio $\alpha \xi_{\mathrm{e}} / \xi_{\mathrm{t}} \quad 0.5$, the plot of Fig. 3a is obtained; moreover if a coefficient is fixed, e.g. $\xi_{\mathrm{t}} 0.05$, while the other is modified, the diagram of
Fig. $3 b$ is drawn. It is seen that a proportional increasing of damping (Fig. 3a) has a stabilization effect on the Hopf linear instability mechanism, but it is uneffective on the double-zero point. In contrast, an additional amount of extensional damping for a fixed torsional damping (Fig. 3b) has a stabilization effect at low stiffness ratios $\kappa$, and an unstabilization effect at high stiffness values. Accordingly, the double-zero point moves along the divergence boundary by visiting the portion marked in Fig. 3b, for which the point $U$ is an upper-bound. Indeed, when $\xi_{\mathrm{e}} \rightarrow 0$, no double-zero bifurcation can take place, as it is confirmed by the fact the two branches of the $I_{1}$-curve (see Fig. 2) approach each other and cross at $U$.

In Fig. 4 the right and left eigenvectors at point $D, H$ and $Z$ of Fig. 2, are displayed.

\subsection{Codimension-1 bifurcations}

The postcritical behaviour of the beam is then analyzed for codimension-1 bifurcations. Eq. (17a) governs the
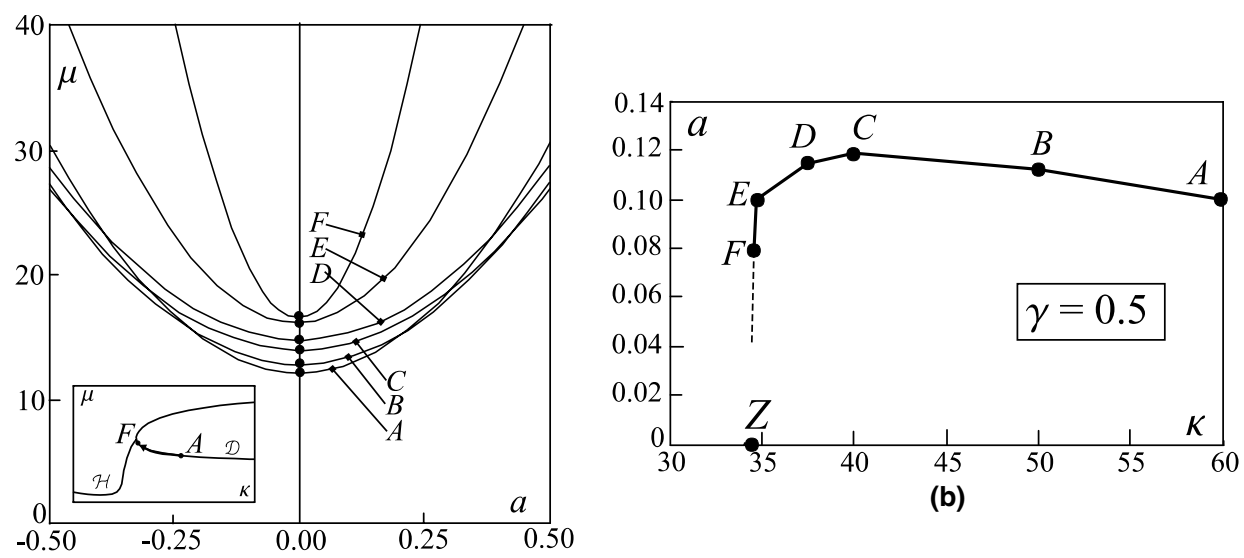

(a)

Fig. 5. (a) Bifurcation diagram around different divergence points along $\mathcal{D}$, of abscises: $\kappa_{A}=60, \kappa_{B}=50, \kappa_{C}=40, \kappa_{D}=37.5, \kappa_{E}=35, \kappa_{F}=34.82$; (b) amplitude $a$ vs the stiffness ratio $\kappa$.

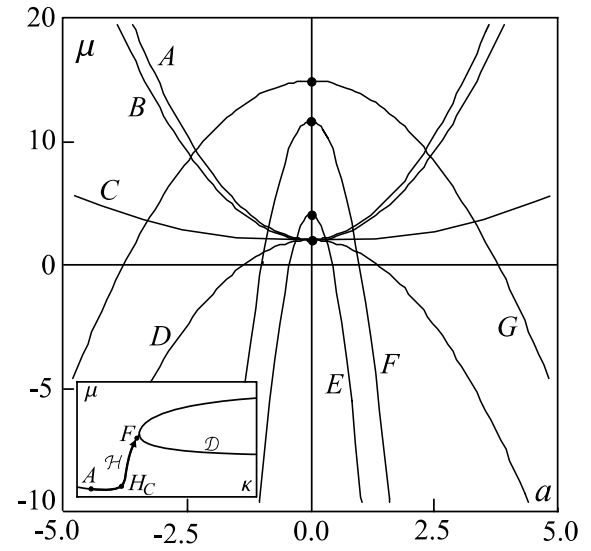

(a)

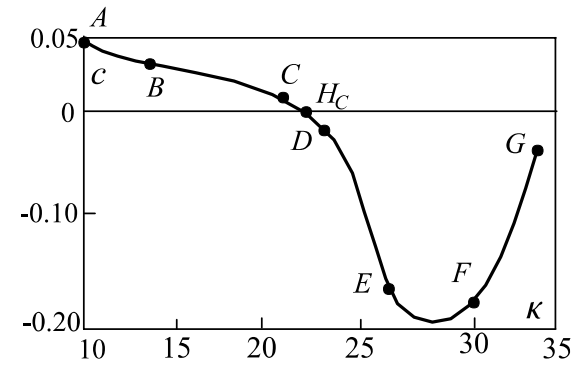

(b)

Fig. 6. (a) Bifurcation diagram around different Hopf points along $\mathcal{H}$, of abscises: $\kappa_{A}=12.04, \kappa_{B}=15.43, \kappa_{C}=21.57, \kappa_{D}=23.16, \kappa_{E}=26.23, \kappa_{F}=30.08$, $\kappa_{G}=33.10 ; \alpha=\xi_{\mathrm{e}} / \xi_{\mathrm{t}}=0.5, \xi_{\mathrm{t}}=0.05 ;$ (b) curvature $c$ of the bifurcated path vs the stiffness ratio $\kappa$. 
dynamic around a divergence point. Steady-state solutions $a$ const $\neq 0$ satisfy the algebraic equation $\mu \quad \mu_{0}-\left(c_{3}\right)$ $\left.c_{1 \gamma}\right) a^{2}$. The equilibrium paths are plotted in Fig. 5a for bifurcations occurring at different points $A F$ along the divergence curve, monotonically approaching $Z$. It is found that the postcritical behaviour is overcritical (stable); however, the curvature of the bifurcated paths does not changes monotonically with the bifurcation point, but decreases from $A$ to $C$ (i.e. nonlinearities are weaker in this range) and increases from $C$ to $F$ (i.e. nonlinearities are stronger in this range). This behaviour is also appreciated in Fig. 5b, where the amplitude $a$ is plotted vs the stiffness $\kappa$ for a fixed value of the parameter $\gamma$.

A different behaviour is found around Hopf bifurcation, governed by Eq. (17b). The limit cycles amplitudes satisfy the equation $\mu \quad \mu_{0}-\left[\operatorname{Re}\left(C_{3}\right) / 4 \operatorname{Re}\left(C_{1 \gamma}\right)\right] a^{2}$; they are plotted in Fig. 6a for bifurcation points $A$ to $G$ monotonically approaching $Z$ along $\mathcal{H}$. The bifurcation is found to be super-critical (stable) far from the double-zero point $Z$ and sub-critical (unstable) close to $Z$; the curvature $c$ of

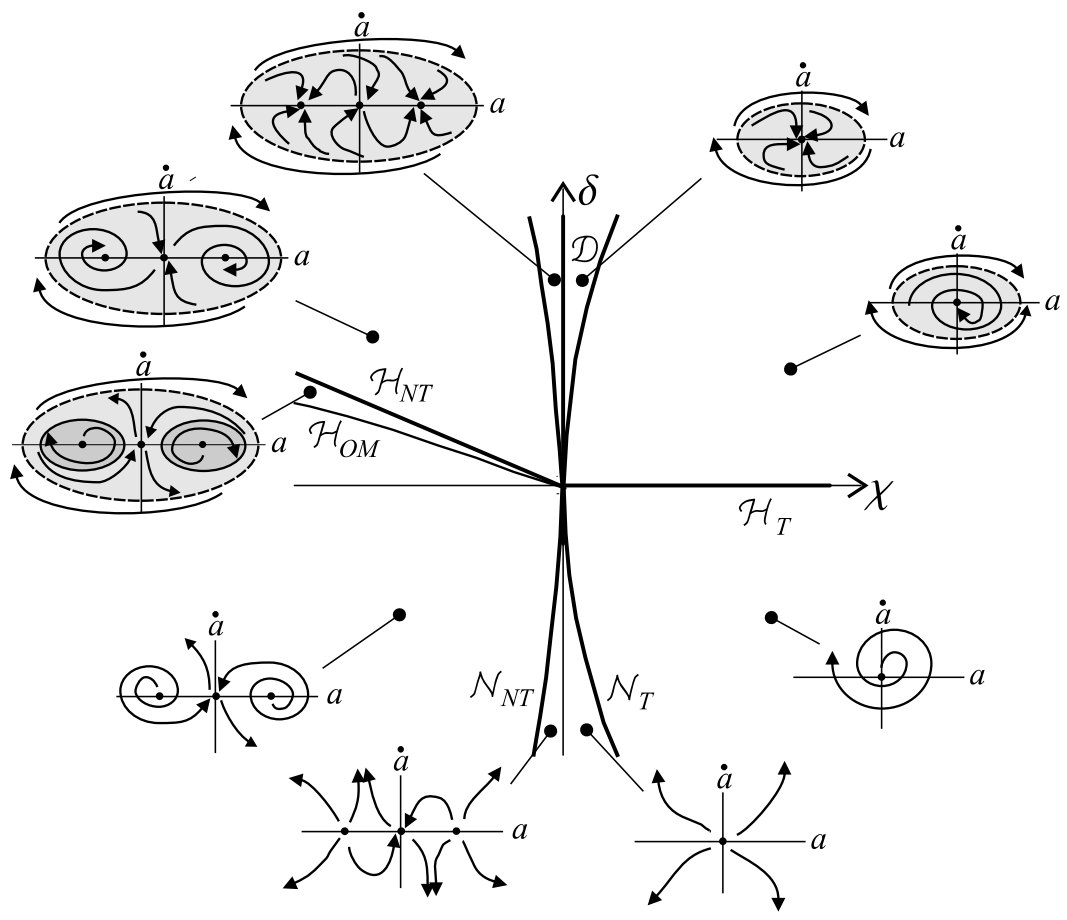

(a)

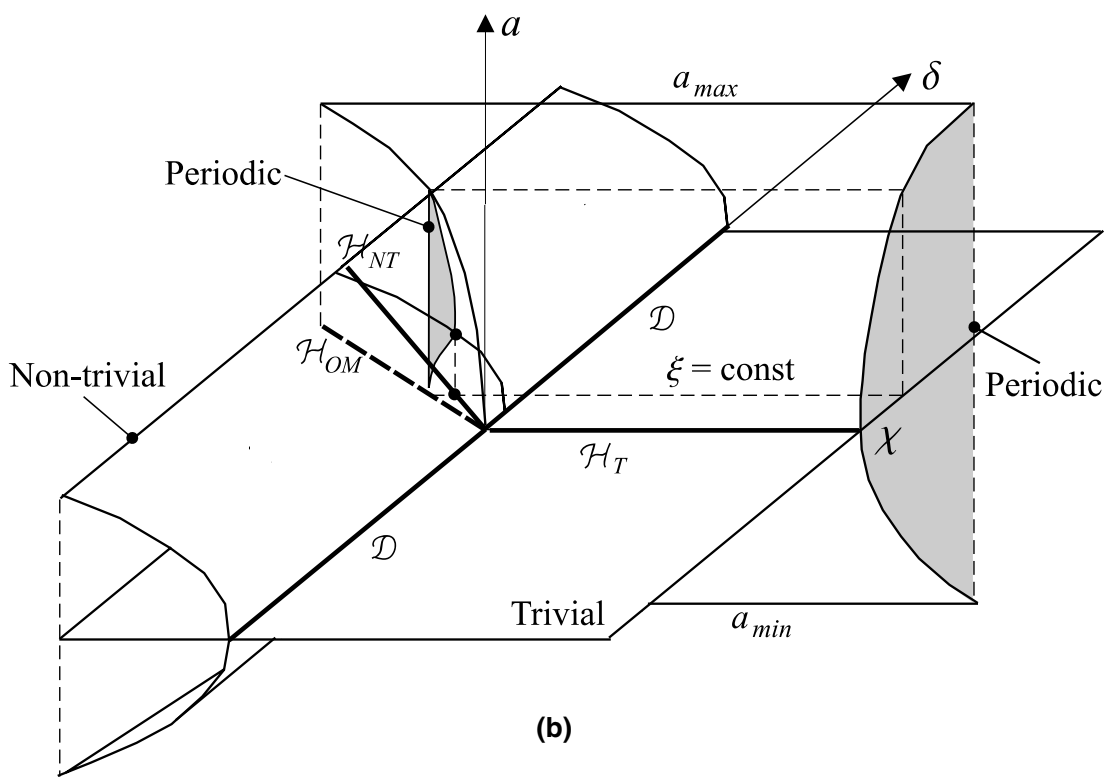

Fig. 7. Bifurcation diagram around a double zero points: (a) unfolding parameter plane and phase plane sketches; (b) equilibriums and limit cycles amplitude vs the unfolding parameters. 
the bifurcation diagram vanishes at point $H_{C}$ marked in Fig. 2 on the Hopf boundary $\mathcal{H}$. This behaviour is also displayed in Fig. 6 b.

\subsection{Codimension-2 bifurcation}

The previous solutions are valid for codimension-1 bifurcations only, and break down close to the double-zero point $Z$. In this region the system behaviour is governed by the bifurcation equation (21), which is here rewritten in the form of a one dof-system equation:

$\ddot{a}=\chi a+\delta \dot{a}+c_{3} a^{3}+e_{3} a^{2} \dot{a}$

In it, $\chi:=-\left(c_{1 \beta} \beta+c_{1 \gamma} \gamma\right), \delta:=-\left(e_{1 \beta} \beta+e_{1 \gamma} \gamma\right)$ are unfolding parameters, assuming the meaning of a stiffness and a damping coefficient respectively, and are linear combinations of the physical parameters $\gamma \quad \mu-\mu_{0}$ and $\beta \quad \kappa-\kappa_{0}$. Their geometrical meaning is shown in Fig. 2, as oblique coordinates measured from $Z$ along the tangents at $\mathcal{H}$ and $\mathcal{D}$ at the double-zero point, respectively. An analysis of Eq. (22) leads to the (qualitative) bifurcation diagram of Fig. 7a and $\mathrm{b}$. The $\delta$-axis is a divergence boundary, while the positive $\chi$-axis is a Hopf boundary for the trivial solution (here denoted by $\mathcal{H}_{\mathrm{T}}$ ). Along the Hopf boundary an under-critical bifurcation occurs, and an unstable limit cycle arises (large cycles in Fig. 7a, in which the system oscillates around the trivial solution). The cycle exists in the angular sector bounded by the $\mathcal{H}_{\mathrm{T}}$ and $\mathcal{H}_{\mathrm{OM}}$ straight lines. Along the divergence boundary a pitchfork bifurcation takes place, for which two nontrivial equilibria emerge, describing symmetrical buckled configurations of the beam. The nontrivial equilibria exist in the half-plane $\chi<0$; they are stable above the straight line $\mathcal{H}_{\mathrm{NT}}$ and unstable below this line. At $\mathcal{H}_{\mathrm{NT}}$ the nontrivial solution undergoes a Hopf bifurcation from

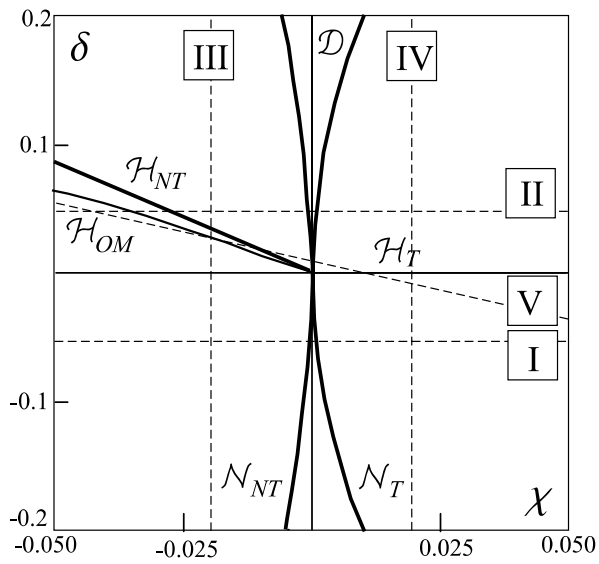

(a)

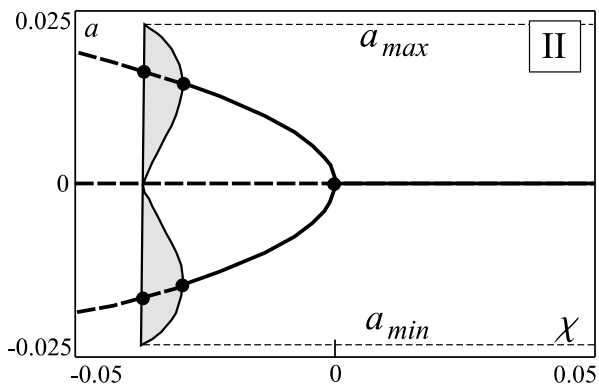

(c)

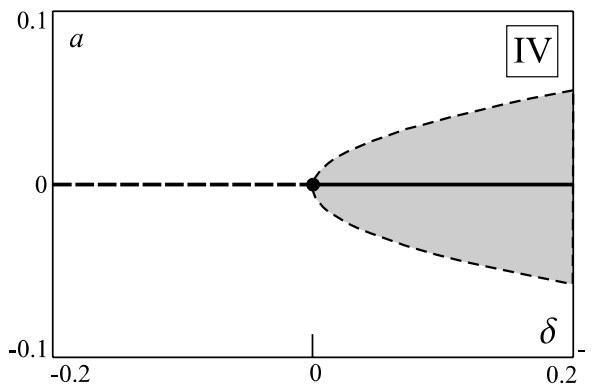

(e)

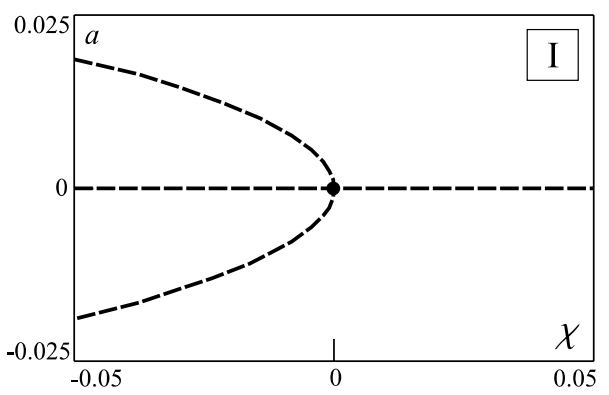

(b)

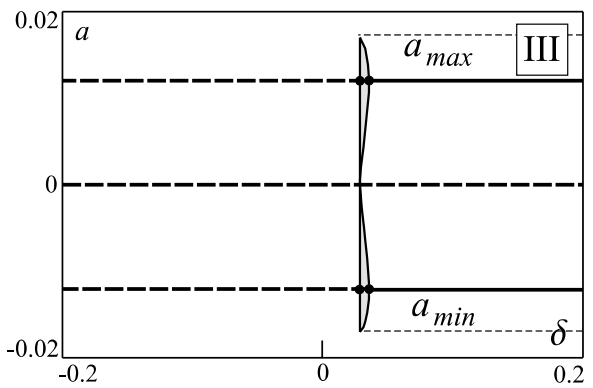

(d)

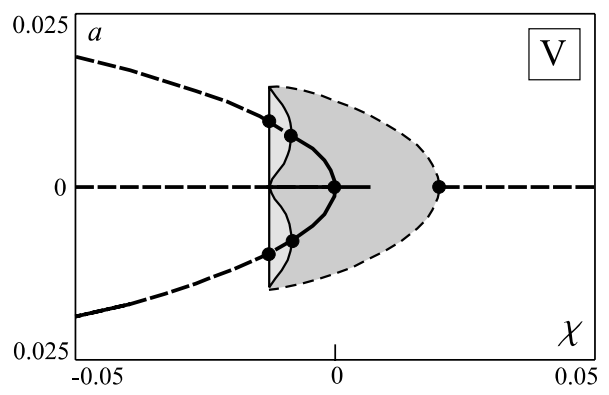

(f)

Fig. 8. Sections of the bifurcation diagram of Fig. 7b: (a) load paths; (b f): sections (I) (V). 
which two stable limit cycles arise (small cycles in Fig. 7a, in which the system oscillates around a buckled configuration). However, they only exist in a small region of the plane, bounded by the straight lines $\mathcal{H}_{\mathrm{NT}}$ and $\mathcal{H}_{\mathrm{OM}}$. Indeed, at $\mathcal{H}_{\mathrm{OM}}$, a homoclinic bifurcation takes place, caused by the multiple collision between the (small) limit cycle, the saddle point at the origin, and the large cycle. In conclusion, stable solutions exist only in the region above the straight lines $\mathcal{H}_{\mathrm{T}}$ and $\mathcal{H}_{\mathrm{OM}}$ of the plane: the trivial equilibrium for $\chi>0, \delta>0$; two stable buckled equilibria between $\mathcal{H}_{\mathrm{NT}}$ and $\mathcal{D}$; the small cycles between $\mathcal{H}_{\mathrm{NT}}$ and $\mathcal{H}_{\mathrm{OM}}$. In addition to the previously defined boundaries, two further curves, $\mathcal{N}_{\mathrm{T}}$ and $\mathcal{N}_{\mathrm{NT}}$, organize the bifurcation diagram, which are loci of (nilpotent) systems possessing two coincident eigenvalues.

Some sections of the bifurcation diagram of Fig. 7b, as obtained by Auto ${ }^{\circledR}$, are displayed in Fig. 8. Five straight paths (I V) have been chosen in Fig. 8a, and the relevant amplitude parameter curves shown in Fig. $8 \mathrm{~b} \mathrm{f}$. Along path I, for decreasing $\chi$ 's, a pitchfork bifurcation is encountered at $\mathcal{D}$. Along path II the pitchfork bifurcation is encountered at $\mathcal{D}$, then the Hopf bifurcation at $\mathcal{H}_{\mathrm{NT}}$, and finally the homoclinic bifurcation at $\mathcal{H}_{\mathrm{OM}}$ (where the cycles collide with the trivial solution and the large cycle). Path III proves the existence of the small limit cycles between $\mathcal{H}_{\mathrm{NT}}$ and $\mathcal{H}_{\mathrm{OM}}$. Path IV displays the large limit cycles. Path $\mathrm{V}$ clearly shows all bifurcations, in particular the simultaneous collision among the cycles and the saddle points.

\section{Conclusions}

The efficiency of the multiple scale method in obtaining reduced-order models of infinite-dimensional systems has been shown. The method consists in the following steps:

(1) The linear differential equation and boundary conditions, are considered. The adjoint differential equation and boundary conditions are derived from the bilinear identity.

(2) The linear stability boundaries of the trivial equilibrium position are analyzed in the parameter space. Both right and left eigenvectors are evaluated at the critical points. If the operator is defective, i.e. if its eigenvectors do not form a complete set, then generalized eigenvectors must be evaluated (said to form a Keldysh chain), similarly to the eigenvectors of an algebraic operator (said to form a Jordan chain).

(3) The state variables are then expanded in series of a perturbation parameter $\varepsilon$; the bifurcation parameters are scaled so that they first appear at the first meaningful order; several independent time-scales are introduced; the perturbation equations are derived by collecting terms of the same $\varepsilon$-order.

(4) The perturbation equations are solved in sequence, which calls for the right-hand side to be orthogonal to the left eigenvectors. These solvability conditions supply amplitude equations on different time-scales.
(5) By recombining the amplitude equations and returning to the true time-scale, the bifurcation equations capturing the asymptotic dynamics of the system are recovered. They are found to be already in normal form.

The procedure has been illustrated for a continuous model of a planar beam, elastically restrained and damped at one end, loaded by a follower force. The relevant bifurcation equations have been numerically solved, and the whole scenario displayed in the parameter space.

\section{Acknowledgements}

This research was supported by the Italian Ministry for Universities and Scientific Research MIUR (Cofin 2003-04 funds).

\section{Appendix}

The coefficients appearing in the bifurcation equation (17a) are:

$$
\begin{aligned}
& c_{1 \gamma}=\gamma a \int_{0}^{1} 2 \varphi^{\prime \prime} \psi \mathrm{d} s \\
& c_{3}=-\left[n_{2}\left(\varphi^{3}\right) \psi_{B}+n_{3}\left(\varphi^{3}\right) \psi_{B}^{\prime}+\int_{0}^{1} n_{1}\left(\varphi^{3}\right) \psi \mathrm{d} s\right]
\end{aligned}
$$

while the coefficients of then bifurcation equation (17b) assume the form:

$$
\begin{aligned}
& C_{1 \gamma}=\int_{0}^{1} 2 \varphi^{\prime \prime} \psi \mathrm{d} s ; \\
& C_{2}=-\left[n_{2}\left(\varphi^{2} \varphi\right) \psi_{B}+n_{3}\left(\varphi^{2} \varphi\right) \psi_{B}^{\prime}+\int_{0}^{1} n_{1}\left(\varphi^{2} \varphi\right) \psi \mathrm{d} s\right]
\end{aligned}
$$

where $n_{i} \cdot(\cdot)(i \quad 1,2,3)$ are the nonlinear cubic terms of Eq. (15b) and $\varphi$ and $\psi$ are the right and the left eigenfunctions, respectively.

Coefficients in Eq. (21) take the following forms:

$$
\begin{aligned}
& c_{1 \beta}=-\varphi_{1 B} \psi_{2 B}, \quad c_{1 \gamma}=2\left(\varphi_{1 B}^{\prime} \psi_{2 B}-\varphi_{1 B} \psi_{2 B}^{\prime}\right) \\
& c_{3}=-\int_{0}^{1} \psi_{2} n_{1}\left(\varphi_{1}^{3}\right) \mathrm{d} s+\psi_{2 B} n_{2}\left(\varphi_{1}^{3}\right)+\psi_{2 B}^{\prime} n_{3}\left(\varphi_{1}^{3}\right)
\end{aligned}
$$

where $n_{i}(\cdot)(i \quad 1,2,3)$ are the nonlinear cubic terms of Eq. $(19 c)$ and

$$
\begin{aligned}
e_{1 \beta}=- & \left(\int_{0}^{1} \psi_{2} \hat{z}_{\beta} \mathrm{d} s+\xi_{\mathrm{e}} \psi_{2 B} z_{\beta B}+\xi_{\mathrm{t}} \psi_{2 B}^{\prime} z_{\beta B}^{\prime}+\psi_{2 B} \varphi_{2 B}\right) \\
e_{1 \gamma}=- & \left(\int_{0}^{1} \psi_{2} \hat{z}_{\gamma} \mathrm{d} s+\xi_{\mathrm{e}} \psi_{2 B} z_{\gamma B}+\xi_{\mathrm{t}} \psi_{2 B}^{\prime} z_{\gamma B}^{\prime}-2 \psi_{2 B} \varphi_{2 B}^{\prime}+2 \psi_{2 B}^{\prime} \varphi_{2 B}\right) \\
e_{3}=- & {\left[\int_{0}^{1} \psi_{2}\left(\hat{z}_{a}-n_{1}\left(\varphi_{1}^{2} \varphi_{2}\right)\right) \mathrm{d} s+\xi_{\mathrm{e}} \psi_{2 B} z_{a B}+\xi_{\mathrm{t}} \psi_{2 B}^{\prime} z_{a B}^{\prime}\right.} \\
& \left.-\psi_{2 B} n_{2}\left(\varphi_{1}^{2} \varphi_{2}\right)-\psi_{2 B}^{\prime} n_{3}\left(\varphi_{1}^{2} \varphi_{2}\right)\right]
\end{aligned}
$$


where $n_{i}(\cdot)(i=1,2,3)$ are the nonlinear cubic terms of Eq. (19d). Moreover, $\varphi_{1}$ and $\varphi_{2}$ are the proper and the generalized right eigenfunctions respectively and $\psi_{2}$ is the proper left eigenfunction. Quantities $z$ and $\hat{z}$ are particular solutions of Eq. (19c) (see [8] for more details).

\section{References}

[1] Steindl A, Troger H. Methods for dimension reduction and their application in nonlinear dynamics. Int J Solids Struct 2001;38: 2131-47.

[2] Nayfeh AH. Reducted-order models of weakly nonlinear spatially continuous systems. Nonlin Dyn 1998;16:105-25.

[3] Nayfeh AH, Lacarbonara W. On the discretization of spatially continuous systems with quadratic and cubic nonlinearities. JSME Int J 1998;41:510-31.

[4] Rega G, Lacarbonara W, Nayfeh AH, Chin C. Multiple resonances in suspended cables: direct versus reduced-order models. Int J NonLin Mech 1999;34:901-24.
[5] Luongo A, Paolone A, Di Egidio A. Sensitivity and linear stability analysis around a double zero eigenvalues. AIAA J 2000;38(4): $702-10$.

[6] Luongo A, Di Egidio A, Paolone A. Multiple time scale analysis for bifurcation from a multiple-zero eigenvalue. AIAA J 2003;41(6): $1143-50$.

[7] Luongo A, Di Egidio A, Paolone A. Multiple scale bifurcation analysis for finite-dimensional autonomous systems. Recent research developments in sound \& vibration, vol. 1. Kerala, India: Transworld Research Network; 2002. p. 161-201.

[8] Luongo A, Di Egidio A. Bifurcation equations through multiplescales analysis for a continuous model of a planar beam. Nonlin Dyn 2005;41(1-3):171-90.

[9] Nayfeh AH. Introduction to perturbation techniques. New York: Wiley-Interscience; 1991.

[10] Luongo A. Eigensolutions sensitivity for nonsymmetric matrices with repeated eigenvalues. AIAA J 1993;31:1321-8.

[11] Keldysh MV. On eigenvalues and eigenfunctions of certain classes of not self-adjoint equations. Doklady Akademii Nauk SSRR 1951; 77(1):11-4 [in Russian]. 